\title{
Three-Dimensional Flexible Endoscopy Can Facilitate Efficient and Reliable Endoscopic Hand Suturing: An ex-vivo Study
}

\author{
Jun Omori, Osamu Goto, Kazutoshi Higuchi, Takamitsu Umeda, Naohiko Akimoto, Masahiro Suzuki, Kumiko Kirita, Eriko \\ Koizumi, Hiroto Noda, Teppei Akimoto, Mitsuru Kaise and Katsuhiko Iwakiri
}

Department of Gastroenterology, Nippon Medical School, Graduate School of Medicine, Tokyo, Japan

Background/Aims: Three-dimensional (3D) flexible endoscopy, a new imaging modality that provides a stereoscopic view, can facilitate endoscopic hand suturing (EHS), a novel intraluminal suturing technique. This ex-vivo pilot study evaluated the usefulness of $3 \mathrm{D}$ endoscopy in EHS.

Methods: Four endoscopists (two certified, two non-certified) performed EHS in six sessions on a soft resin pad. Each session involved five stitches, under alternating 3D and two-dimensional (2D) conditions. Suturing time (sec/session), changes in suturing time, and accuracy of suturing were compared between $2 \mathrm{D}$ and $3 \mathrm{D}$ conditions.

Results: The mean suturing time was shorter in $3 \mathrm{D}$ than in $2 \mathrm{D}(9.8 \pm 3.4 \mathrm{~min} / \mathrm{session}$ vs. $11.2 \pm 5.1 \mathrm{~min} / \mathrm{session})$ conditions and EHS was completed faster in $3 \mathrm{D}$ conditions, particularly by non-certified endoscopists. The suturing speed increased as the $3 \mathrm{D}$ sessions progressed. Error rates (failure to grasp the needle, failure to thread the needle, and puncture retrial) in the 3D condition were lower than those in the $2 \mathrm{D}$ condition, whereas there was no apparent difference in deviation distance.

Conclusions: 3D endoscopy may contribute to increasing the speed and accuracy of EHS in a short time period. Stereoscopic viewing during $3 \mathrm{D}$ endoscopy may help in efficient skill acquisition for EHS, particularly among novice endoscopists. Clin Endosc 2020;53:334-338

Key Words: Endoscopic suturing; Learning curve; Three-dimensional endoscopy

\section{INTRODUCTION}

Endoscopic hand suturing (EHS) is a novel suturing method that allows optimal and secure intraluminal suturing. ${ }^{1,2}$ In EHS for mucosal defects, the mucosal rim of the defect is continuously and linearly sutured using a transorally delivered needle and suture. Although EHS is expected to be a promising technique for the development of various advanced therapeutic endoscopies, it requires advanced technical skills and significant experience in puncturing the mucosa using

Received: October 29, 2019 Revised: February 15, 2020

Accepted: March 10, 2020

Correspondence: Osamu Goto

Department of Gastroenterology, Nippon Medical School, Graduate School of Medicine, 1-1-5, Sendagi, Bunkyo-ku, Tokyo 113-8603, Japan

Tel: +81-3-3822-2131, Fax: +81-3-5814-6289, E-mail: o-goto@nms.ac.jp

ORCID: https://orcid.org/0000-0002-1039-6323

(c) This is an Open Access article distributed under the terms of the Creative Commons Attribution Non-Commercial License (http://creativecommons.org/ licenses/by-nc/3.0) which permits unrestricted non-commercial use, distribution, and reproduction in any medium, provided the original work is properly cited. the needle, optimally grasping the needle, and tightening the thread using a flexible endoscope; therefore, how to efficiently obtain these necessary skills is an issue to be addressed.

Technological advances in laparoscopic surgery have enabled the creation of stereoscopic videos or three-dimensional (3D) images from conventional two-dimensional (2D) video images and have led to favorable results in the clinical settings. ${ }^{3,4}$ Recently, 3D flexible endoscopy has also been described and is expected to be useful for both endoscopic diagnosis $^{5,6}$ and treatment. ${ }^{7-9}$ Thus, we hypothesized that 3D endoscopy can reliably enhance EHS performance compared with conventional $2 \mathrm{D}$ endoscopy and verified the usefulness of $3 \mathrm{D}$ endoscopy in EHS in an ex-vivo study.

\section{MATERIALS AND METHODS}

\section{Participants}

Four endoscopists (two certified and two non-certified) without prior EHS experience, were selected and provided 
brief instruction by an EHS expert. A certified endoscopist was defined as a doctor with a license of specialist as authorized by the Japan Gastroenterological Endoscopy Society. Each endoscopist performed EHS alternately under 2D or 3D conditions, with six sessions per condition sequentially, with one assistant having minimal EHS experience. The participants had not received any prior training. Written informed consent was provided by the participants prior to study initiation.

\section{Endoscopy system}

The endoscopy system used was identical to that used in surgery, which mainly included an EVIS EXERA III Video System Center, equipped with a 3D Visualisation Unit (CV190; Olympus Co., Ltd., Tokyo, Japan) and EVIS EXERA III Video Processor (3DV-190; Olympus). In these systems, switching from the $2 \mathrm{D}$ to the $3 \mathrm{D}$ mode and vice versa can be accomplished by pushing a button on the head of the endoscope. The $3 \mathrm{D}$ images were visualized using a 3D monitor and 3D glasses, as described elsewhere. ${ }^{7-9}$

\section{Endoscopic hand suturing settings and procedures}

We prepared eight urethane pads designed for surgical skin suturing training (AS ONE Co., Osaka, Japan). Each pad could accommodate six sutures, and each suturing area had 10 markings: five each on the right and left rows (Fig. 1A). The interval between successive markings was set at $5 \mathrm{~mm}$. One pad was used per one condition (2D or $3 \mathrm{D}$ ); thus, each participant used two pads. The pads were placed in a multipurpose training box for flexible endoscopy using EHS.

EHS was performed using a GIF-Y0080 3D scope (Olympus) equipped with a prototype flexible needle holder (Olympus) and 15-cm, 3-0 absorbable barbed V-Loc 180 (VLOCL0604) sutures (Covidien, Mansfield, MA, USA), as described elsewhere. ${ }^{1,2}$ Briefly, a needle with a knot at the tail was inserted from the markings on the right row and pulled out from the markings on the left row (Supplementary video 1), and the sutures were placed continuously and linearly from the distal to the proximal side (Fig. 1B). Any slack in the thread was appropriately tightened by directly grasping the thread with the needle holder. All procedures were recorded as videos.

\section{Assessments and outcome measures}

While reviewing the videos, we estimated the suturing time (sec/session) and number of errors (failure to grasp the needle, failure to grasp the thread, and puncture repetition). Next, the sutured pad was used to assess deviation distance, measured as the interval between the actual insertion/exit site and the marking where the needle should have passed.

The primary outcome measure was suturing time under

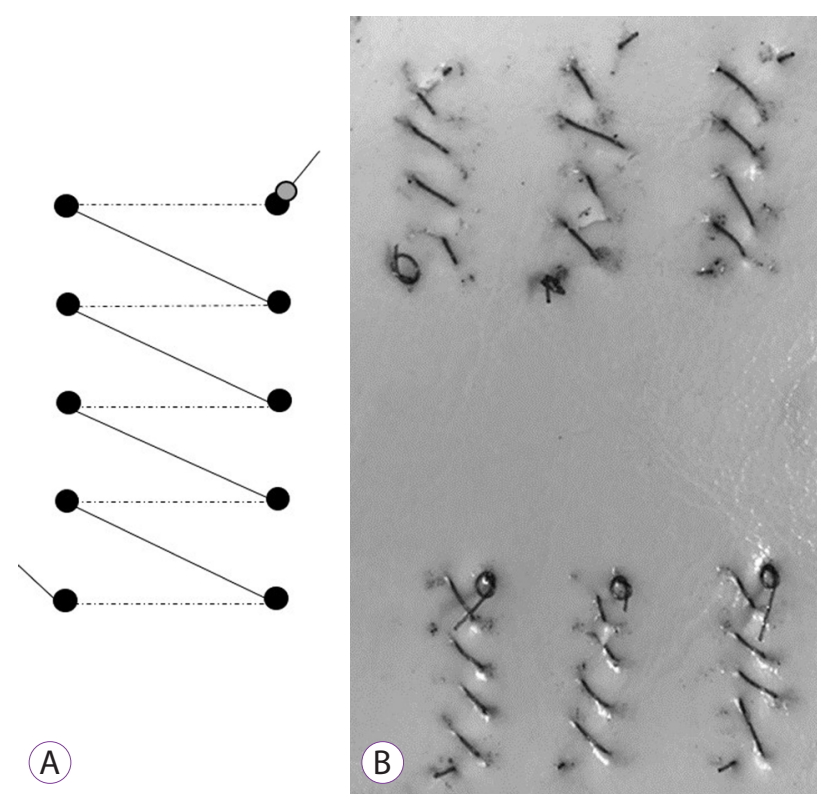

Fig. 1. Ex-vivo settings of endoscopic hand suturing. (A) Locations of the markings on the urethane pad. Five markings at $5-\mathrm{mm}$ intervals are linearly placed in both the right and left rows. (B) The pad after suturing. The suture needle is inserted from the marking point on the right row and pulled out from the marking point on the left row; this suturing is repeated five times per session.

two conditions (2D and 3D). The suturing time for each endoscopist, changes in suturing time, deviation distance, and error rate were also evaluated. Finally, subjective assessments among the endoscopists (degree of learning, interest, satisfaction, self-confidence, difficulty, and eye strain) after the EHS procedure were also performed using a visual analog scale (VAS) scale, with scores ranging from 1 to 4 points (1: very bad, 2: bad, 3: good, 4: very good).

\section{Statistical analyses}

Because this phase I pilot study lacked a statistically calculated sample size, the obtained data were shown with 95\% confidence intervals (CIs). We analyzed the suturing time and error rates between the $2 \mathrm{D}$ and $3 \mathrm{D}$ groups using nonparametric statistic methods (Mann-Whitney $U$ tests). Analyses were performed using the SPSS statistical software package version 25 (IBM Co., New York, NY, USA).

\section{RESULTS}

Of 48 total sessions by four participants in the $2 \mathrm{D}$ and $3 \mathrm{D}$ conditions, EHS was successfully completed in all except for one 2D session (47/48 sessions; 98\%) due to unexpected thread disconnection. We omitted data from this incomplete session during analyses and did not add a compensatory ses- 


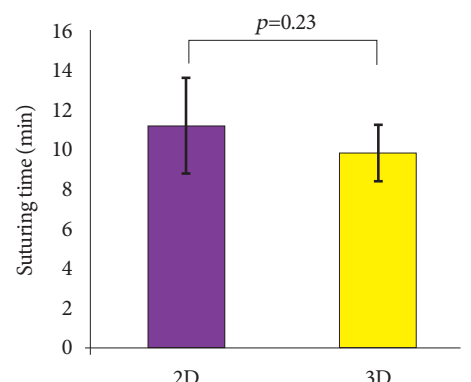

(A)

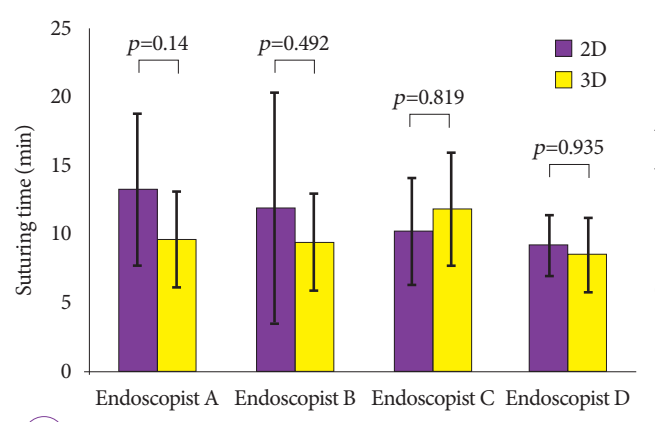

(B)

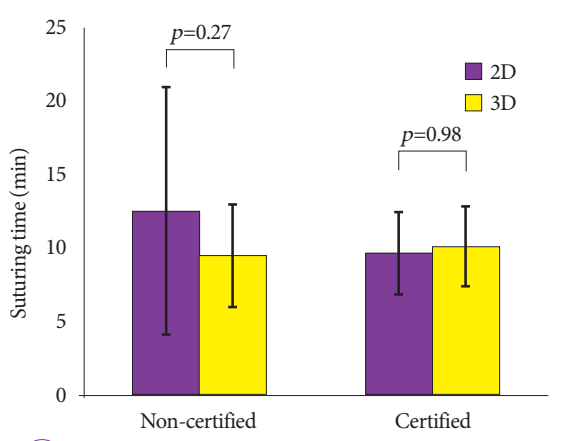

(C)

Fig. 2. Suturing time during endoscopic hand suturing (EHS). (A) Comparison of suturing time (min/session) between two-dimensional (2D) and three-dimensional (3D) endoscopy showing a shorter time in 3D. (B) Comparison of suturing time for each endoscopist. (C) Among non-certified participants, EHS is completed faster in $3 \mathrm{D}$ than in 2D. Bar: $95 \%$ confidence interval.

$2 \mathrm{D}$

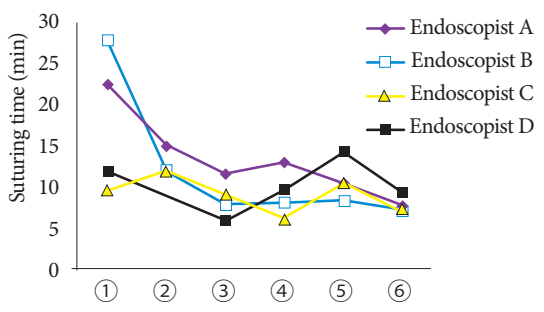

(A)

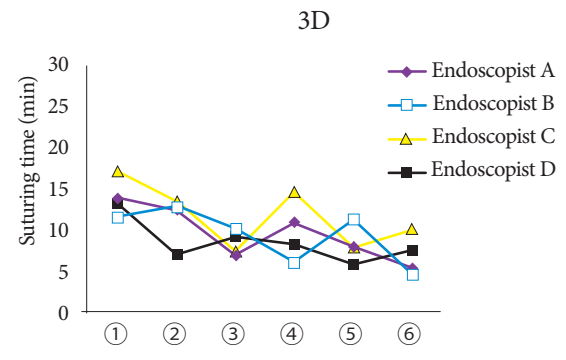

(B)

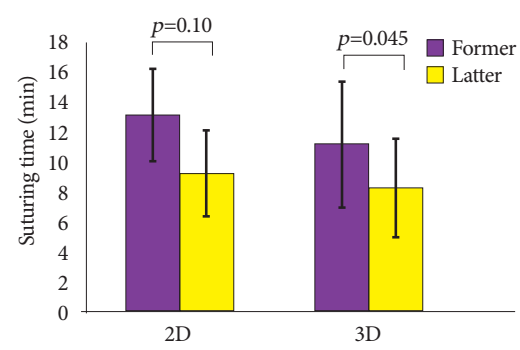

(C)

Fig. 3. Changes in suturing time. (A) Under two-dimensional (2D) conditions, the suturing time per session tends to decrease as the study proceeded. (B) The time also decreases in the three-dimensional (3D) condition, although the magnitude of change is less than that seen under $2 \mathrm{D}$ conditions. (C) Comparison of suturing times in the former and latter halves of the suture sessions showing a reduced time in the 3D condition. Bar: 95\% confidence interval.

sion to avoid a possible learning effect from the incomplete session.

The mean suturing time was $9.8 \pm 3.4 \mathrm{~min} / \mathrm{session}(95 \%$ $\mathrm{CI}, 8.4-11.2)$ in $3 \mathrm{D}$, which was shorter than in that in $2 \mathrm{D}$ $(11.2 \pm 5.1 \mathrm{~min} /$ session [95\% CI, 8.9-13.4]), but this difference was not significant ( $p=0.23$ ) (Fig. 2A). No participants showed a significant increase in their suturing speed in $3 \mathrm{D}$ compared to that in 2D (Fig. 2B). Similarly, no significant difference in suturing time was seen between the $3 \mathrm{D}$ and $2 \mathrm{D}$ conditions between certified and non-certified endoscopists (10.1 min/ session in $3 \mathrm{D}$ vs. $9.6 \mathrm{~min} /$ session in $2 \mathrm{D}, p=0.98$; non-certified, $9.5 \mathrm{~min} / \mathrm{session}$ in $3 \mathrm{D}$ vs. $12.5 \mathrm{~min} / \mathrm{session}$ in $2 \mathrm{D}, p=0.27$ ) (Fig. 2C).

Fig. 3 shows the changes in suturing time. All participants tended to suture faster under both conditions as their experience with EHS increased during the course of this study. Specifically, the suturing time decreased with less variation in $3 \mathrm{D}$ than in $2 \mathrm{D}(\mathrm{R}=0.65$ in $3 \mathrm{D}$ and 0.56 in $2 \mathrm{D})$ (Fig. $3 \mathrm{~A}$, $B)$. Furthermore, the mean suturing time in the first and last three sessions were 13.2 and $9.3 \mathrm{~min} /$ session in $2 \mathrm{D}(p=0.10)$ and 11.3 and $8.4 \mathrm{~min} / \mathrm{session}$ in $3 \mathrm{D}(p=0.045)$, with the latter showing a significant reduction in the suturing time (Fig. 3C).

There was no apparent difference in deviation distance be-
Table 1. Outcomes of Technical Accuracy in Endoscopic Hand Suturing

\begin{tabular}{lccc}
\hline & 2D & 3D & $p$-value \\
\hline Deviation distance $(\mathrm{mm})$ & $11.1 \pm 4.1$ & $11.4 \pm 5.6$ & 0.84 \\
Error rates (times) & & & \\
Fail to grasp of the needle & $3.0 \pm 2.9$ & $2.2 \pm 2.1$ & 0.29 \\
Fail to grasp of the thread & $0.8 \pm 1.5$ & $0.3 \pm 0.5$ & 0.11 \\
Retry of the puncture & $2.0 \pm 2.1$ & $1.5 \pm 1.1$ & 0.28 \\
Subjective assessments & & & \\
Degree of learning & $3.0 \pm 0.0$ & $3.3 \pm 0.5$ & 0.39 \\
Interest & $2.8 \pm 0.5$ & $3.3 \pm 1.0$ & 0.39 \\
Satisfaction & $2.5 \pm 0.6$ & $3.3 \pm 0.5$ & 0.097 \\
Self-confidence & $2.5 \pm 0.6$ & $3.3 \pm 0.5$ & 0.097 \\
Difficulty & $2.3 \pm 0.5$ & $2.5 \pm 1.0$ & 0.67 \\
Eye strain & $3.0 \pm 0.0$ & $2.8 \pm 0.5$ & 0.39 \\
\hline
\end{tabular}

$2 \mathrm{D}$, two-dimensional condition; $3 \mathrm{D}$, three-dimensional condition. 
tween the two conditions (Table 1). However, the error rates (failure to grasp the needle, failure thread the needle, and puncture retrial) in the 3D group were lower than those in the $2 \mathrm{D}$ group (Table 1). Concerning subjective assessments, we found no significant differences between the $2 \mathrm{D}$ and $3 \mathrm{D}$ groups in the degree of learning ( 3 vs. $3.25, p=0.39$ ), interest ( 2.75 vs. $3.25, p=0.39$ ), satisfaction ( 2.5 vs. $3.25, p=0.097$ ), self-confidence ( 2.5 vs. $3.25, p=0.097$ ), difficulty ( 2.25 vs. 2.5 , $p=0.67$ ), and eye strain ( 3 vs. $2.75, p=0.39$ ) (Table 1 ).

\section{DISCUSSION}

This ex-vivo pilot study investigated the usefulness of 3D endoscopy in EHS, a representative advanced endoscopic technique. The results demonstrated that $3 \mathrm{D}$ endoscopy facilitated EHS compared to 2D endoscopy as it reduced suturing time. 3D endoscopy facilitates stereoscopic depth recognition; therefore, endoscopists can easily grasp, set the direction, and insert the needle at an optimal angle in EHS.

Sakata et al. ${ }^{10}$ demonstrated that all laparoscopic tasks were faster in 3D than in 2D, with an approximately $30 \%$ reduction in completion time and that 3D endoscopy appeared to contribute to time savings during endoscopic submucosal dissection in ex-vivo porcine models. ${ }^{8,9}$ We observed a $12 \%$ reduction in suturing time in EHS (from 11.2 to $9.8 \mathrm{~min} / \mathrm{session}$ ) in the present study, although the difference was not statistically significant. The lack of significant reduction may be due to the insufficient number of sessions and the difficulty of the task tested, as suturing involves tissue apposition requiring at least two stitches and tightening of the thread; continuing this procedure under flexible endoscopy is complicated and technically demanding. Furthermore, the artificial situation by the use of urethane pads may prevent smooth suturing compared to other conditions such as isolated/live porcine stomachs or even clinical settings. Therefore, the $12 \%$ reduction in suturing time under this condition is acceptable to demonstrate the advantage of 3D in EHS. In one endoscopist, the mean suturing time was shorter in $2 \mathrm{D}$ than that in $3 \mathrm{D}$ because the suture was accidentally cut when a slack part of the suture was pulled too strongly in one 3D session, which required extra time to retry the suturing.

3D endoscopy appears particularly helpful in novice endoscopists. In general, repetitive training in endoscopic procedures accelerates the improvement of these skills, which is also applicable to difficult and complicated techniques including EHS. In this study, by using 3D, even non-certified endoscopists performed EHS in comparable times to those of certified endoscopists. This finding implies that 3D may facilitate tech- nically challenging procedures for less experienced endoscopists. Further study is needed to investigate the potential of 3D endoscopy to make advanced procedures accessible to endoscopists with various degrees of technical skill.

Our data also showed a significant reduction in suturing time in the last three sessions of 3D endoscopy, suggesting a meaningful learning effect and implying that endoscopists can more efficiently acquire suturing skills using $3 \mathrm{D}$ viewing compared with $2 \mathrm{D}$ viewing when EHS is repeatedly performed. A previous report also indicated that robotic surgery improves surgical performance and learning, especially with 3D viewing. ${ }^{11}$ Taken together, these results indicate that the skills required for advanced endoscopic techniques can be obtained more quickly using 3D endoscopy; however, further assessment is needed.

Although the error rates were lower under the 3D condition than those under the 2D condition, we could not determine the improvement in the technical accuracy of EHS due to the lack of clear evidence. The lower error rates may have been because of better depth perception in the 3D view compared to that in the $2 \mathrm{D}$ view, as the former simulates an environment similar to the real world.

We evaluated the subjective assessment of endoscopists using the VAS scale (learning, interest, satisfaction, self-confidence, difficulty, eye strain) and observed no significant differences between the two conditions. However, the relatively high scores for satisfaction and self-confidence in the 3D groups compared to those in the $2 \mathrm{D}$ groups may imply that $3 \mathrm{D}$ endoscopy is more accessible to endoscopists than conventional endoscopy.

The present study has several limitations. First, all participants always started the procedure with the $2 \mathrm{D}$ condition and performed suturing under either $2 \mathrm{D}$ or $3 \mathrm{D}$ alternately per session (1st, 2D; 2nd, 3D; 3rd, 2D; 4th, 3D, etc.). Therefore, a training effect by performing 2D first could be a bias in this study. Second, this was an ex-vivo pilot study under artificially provided conditions with a small number of sessions and participants. A prospective comparative study in clinical settings with a large number of endoscopists and a statistically calculated sample size based on this preclinical pilot study is necessary to validate these preliminary results on the usefulness of 3D endoscopy in EHS.

In conclusion, our data suggest that $3 \mathrm{D}$ endoscopy may efficiently contribute to fast and accurate EHS and may be particularly helpful for novice endoscopists. Further clinical investigations are necessary to validate these results.

\section{Conflicts of Interest}

The flexible needle holder and scissors forceps used in this study were complimentarily provided by Olympus Co., Ltd. 
Author Contributions

Conceptualization: Osamu Goto

Data curation: Jun Omori, Kazutoshi Higuchi, Takamitsu Umeda, Naohiko Akimoto, Masahiro Suzuki

Formal analysis: $\mathrm{JO}$

Funding acquisition: OG

Investigation: JO, Kumiko Kirita, Eriko Koizumi, Hiroto Noda, Teppei Akimoto

Methodology: OG

Project administration: JO, OG

Supervision: OG, Mitsuru Kaise, Katsuhiko Iwakiri

Validation: OG, KK, EK, HN, TA

Writing-original draft: JO

Writing-review\&editing: JO, MK, KI

\section{Supplementary Material}

Video 1. Endoscopic hand suturing in this ex-vivo study. The soft urethane pad is continuously sutured with a barbed suture and a flexible needle holder, from right to left, and from the distal to the proximal direction. A loosened part of the string is appropriately tightened (https://doi. org/10.5946/ce.2019.207.v001)

\section{ORCID}

Jun Omori: https://orcid.org/0000-0002-4375-5070

Kazutoshi Higuchi: https://orcid.org/0000-0003-4386-6288

Takamitsu Umeda: https://orcid.org/0000-0001-6997-8013

Naohiko Akimoto: https://orcid.org/0000-0001-9880-4143

Masahiro Suzuki: https://orcid.org/0000-0002-3260-7654

Kumiko Kirita: https://orcid.org/0000-0003-2960-6690

Eriko Koizumi: https://orcid.org/0000-0002-1767-3696

Hiroto Noda: https://orcid.org/0000-0003-1180-7128

Teppei Akimoto: https://orcid.org/0000-0002-7472-5210

Mitsuru Kaise: https://orcid.org/0000-0002-4593-9527

Katsuhiko Iwakiri: https://orcid.org/0000-0002-5558-6104

\section{REFERENCES}

1. Goto O, Sasaki M, Ishii H, et al. A new endoscopic closure method for gastric mucosal defects: feasibility of endoscopic hand suturing in an ex vivo porcine model (with video). Endosc Int Open 2014;2:E111-E116.

2. Goto O, Sasaki M, Akimoto T, et al. Endoscopic hand-suturing for defect closure after gastric endoscopic submucosal dissection: a pilot study in animals and in humans. Endoscopy 2017;49:792-797.

3. Becker H, Melzer A, Schurr MO, Buess G. 3-D video techniques in endoscopic surgery. Endosc Surg Allied Technol 1993;1:40-46.

4. Fergo C, Burcharth J, Pommergaard HC, Kildebro N, Rosenberg J. Three-dimensional laparoscopy vs 2-dimensional laparoscopy with high-definition technology for abdominal surgery: a systematic review. Am J Surg 2017;213:159-170.

5. Nomura K, Kaise M, Kikuchi D, et al. Recognition accuracy using 3D endoscopic images for superficial gastrointestinal cancer: a crossover study. Gastroenterol Res Pract 2016;2016:4561468.

6. Nomura K, Kaise M, Kikuchi D, et al. Recognition accuracy of tumor extent using a prototype 3D endoscope for superficial gastric tumor: an ex vivo crossover study. Endosc Int Open 2018;6:E652-E658.

7. Kikuchi D, Kaise M, Nomura K, et al. Feasibility study of the three-dimensional flexible endoscope in endoscopic submucosal dissection: an ex vivo animal study. Digestion 2017;95:237-241.

8. Nomura K, Kikuchi D, Kaise M, et al. Comparison of 3D endoscopy and conventional 2D endoscopy in gastric endoscopic submucosal dissection: an ex vivo animal study. Surg Endosc 2019;33:4164-4170.

9. Akizue N, Matsumura T, Maruoka D, et al. Novel three-dimensional imaging system may facilitate gastric endoscopic submucosal dissection procedure: an ex vivo animal study. Endosc Int Open 2018;6:E1431-E1435.

10. Sakata S, Grove PM, Hill A, Watson MO, Stevenson ARL. Impact of simulated three-dimensional perception on precision of depth judgements, technical performance and perceived workload in laparoscopy. Br J Surg 2017;104:1097-1106.

11. Blavier A, Gaudissart Q, Cadière GB, Nyssen AS. Comparison of learning curves and skill transfer between classical and robotic laparoscopy according to the viewing conditions: implications for training. Am J Surg 2007;194:115-121. 